\title{
Performance of 2D-SWE.GE for predicting different stages of liver fibrosis, using Transient Elastography as the reference method
}

\author{
Felix Bende, IoanSporea, Roxana Șirli, Alina Popescu, Ruxandra Mare, Bogdan Miuțescu, \\ Raluca Lupuşoru, Tudor Moga, Corina Pienar
}

Department of Gastroenterology and Hepatology, "Victor Babeș” University of Medicine and Pharmacy Timișoara, Romania

\begin{abstract}
Aim: To evaluate the performance of 2D shear-wave elastography from General Electric (2D-SWE.GE) for the noninvasive assessment of liver fibrosis and to identify liver stiffness (LS) cut-off values for predicting different stages of fibrosis using Transient Elastography (TE) as the reference method. Material and method: We included 331 consecutive subjects with or without chronic hepatopathies in whom LS was evaluated in the same session by means of 2 elastographic techniques: TE and 2D-SWE.GE. Reliable LS measurements were defined for TE as the median value of 10 measurements with a success rate of $\geq 60 \%$ and an interquartile range (IQR) $<30 \%$ and for 2D-SWE.GE as the median value of 10 measurements acquired in a homogenous area and an IQR $<30 \%$. To discriminate between TE fibrosis stages we used the following cut-offs: F2- 7; F3- 9.5 and F4- 12kPa. Results: Reliable LS measurements were obtained in $95.8 \%$ subjects by 2D-SWE.GE, and $94.2 \%$ by TE $(\mathrm{p}=0.44)$. Based on TE cut-off values we divided our cohort into four groups: $\mathrm{F}<2: 30.1 \% ; \mathrm{F}=2: 10.2 \% ; \mathrm{F}=3: 12.2 \%$; $\mathrm{F}=4: 47.5 \%$. A strong correlation was found between the LS values obtained by the 2 methods: $\mathrm{r}=0.83$, $\mathrm{p}<0.0001$.LS values obtained by 2D-SWE.GE were significantly lower than those obtained by TE: $10.14 \pm 4.24 \mathrm{kPa}$ vs. $16.72 \pm 13.4 \mathrm{kPa}(\mathrm{p}<0.0001)$. The best cut-off value for $\mathrm{F} \geq 2, \mathrm{~F} \geq 3$ and for $\mathrm{F}=4$ were $6.7,8.2$ and $9.3 \mathrm{kPa}$. Conclusions: The best 2D-SWE.GE cut-off values for predicting $\mathrm{F} \geq 2, \mathrm{~F} \geq 3$ and $\mathrm{F}=4$ were $6.7,8.2$ and $9.3 \mathrm{kPa}$.
\end{abstract}

Keywords: liver stiffness; shear waves elastography; 2D-SWE.GE; transient elastography; liver fibrosis

\section{Introduction}

Liver fibrosis is a progressive process that develops as a consequence of liver damage in the majority of chronic liver diseases. While there are many causes of liver diseases, the most frequently encountered in daily practice are chronic viral hepatitis ( $\mathrm{B}$ or $\mathrm{C}$ viruses), alcoholic steatohepatitis (ASH), and non-alcoholic fatty liver disease (NAFLD). Other chronic liver diseases, such as autoimmune hepatitis or primary biliary cirrhosis (PBC) are also diagnosed in daily practice.

Received 07.12.2016 Accepted 31.01.2017

Med Ultrason

2017, Vol. 19, No 2, 143-149

Corresponding author: Felix Bende

3 Apro Mihai Martir street, ap. 13

300727 Timișoara, Romania

Phone: +40755353852

E-mail: bendefelix@gmail.com
An accurate staging of liver fibrosis is mandatory, especially for prognosis, disease management, treatment indication and long-term follow-up. Liver biopsy is considered as the "gold-standard" method for fibrosis assessment and for necro-inflammatory activity grading [1], but it has some limitations such as: invasive procedure, potential complications, sampling errors, intra- and inter-observer variability [2-4]. Given these limitations, LB is not always accepted by patients for the assessment of liver disease severity, especially for repetitive evaluation of disease progression, thus, in some countries, the number of liver biopsies seems to decrease [5], while the non-invasive methods for fibrosis assessment are used more and more.

Non-invasive methods for the assessment of liver disease severity are: biological tests [6] and elastographic methods (ultrasound-based elastography and MR elastography). According to the European Federation of 
Societies in Ultrasound in Medicine and Biology (EFSUMB) Guidelines, the ultrasound-based elastographic methods are divided into strain/displacement elastography and shear waves elastography techniques [7]. The last category includes Transient Elastography (TE), point shear wave elastography (VTQ and ElastPQ), and 2Dshear wave elastography (including 2D-SWE and 3DSWE) [7]. In the last years, non-invasive evaluation of liver fibrosis has become more and more popular in daily practice mainly due to constant industry development and to the lack of invasiveness. While the use of liver biopsy is declining [5], new elastographic methods are becoming available, making it difficult to say which one should be used in daily practice [8].

The first elastographic method developed for liver fibrosis assessment was Transient Elastography (TE) [9]. Several meta-analyses have thoroughly validated the method [10-12], which is now accepted by international guidelines $[9,13,14]$. It is commonly used in the evaluation of various liver diseases such as chronic hepatitis $\mathrm{C}$ [12,15], chronic hepatitis B [16,17], NAFLD [18], PBC [19] or in post-transplant patients [20]. Despite the fact that $\mathrm{TE}$ is extensively used in daily practice around the world, it presents several limitations. The main disadvantages are that it cannot be performed in patients with ascites [7], and that it lacks B-mode gray scale ultrasound visualization of the liver. Furthermore, the method's reliability decreases from normal weight to overweight and obese patients [21]; this disadvantage has been partially overcame by the use of the XL probe [22,23]. Previous studies have shown that there are confounding factors that can increase LS values by TE: high levels of aminotransferases [24], extrahepatic cholestasis [25] and liver congestion in heart failure [26]. Also, the machine is quite expensive and needs periodical calibration of the probes.

Other elastographic methods, such as point shear wave elastography (pSWE) and 2D shear wave elastography (2D-SWE) are integrated into ultrasound machines, allowing a real-time visualization of liver structure, the ability to choose the region of interest, avoiding vessels and the liver capsule. Another advantage is their capability to perform other ultrasound investigations such as Doppler examination or Contrast Enhanced Ultrasound.

2D-Shear Wave Elastography (2D-SWE) is an imaging technique which quantifies tissue stiffness by measuring the speed of shear waves induced into the tissue by acoustic push pulses, generating two-dimensional quantitative images of shear-wave speed. Nowadays, the technique of 2D-SWE is implemented on several ultrasound machines from different manufacturers (SuperSonic Imagine, General Electric Healthcare, Toshiba). In the last years, several studies have been published demonstrating that SuperSonic Imagine 2D-SWE (2D-SWE.SSI) is an useful tool for evaluating liver fibrosis, non-inferior to TE [27-29]. To our knowledge, there are no studies that have evaluated 2D-SWE on General Electric Healthcare (2D-SWE.GE) in clinical practice.

The aim of this paper was to identify the cut-off values of liver stiffness (LS) assessed by means of 2D-SWE. GE for predicting different stages of liver fibrosis, using TE, a validated method for liver fibrosis assessment, as the reference method $[9,13,14]$.

\section{Material and methods}

\section{Subjects}

We performed a prospective study which included 331 consecutive adult subjects: 56 healthy volunteers and 275 patients with chronic liver disease. All subjects agreed to undergo elastographic measurements (signed informed consent). The study was approved by the local Ethics Committee and was performed in accordance with the last revised version of the Helsinki Declaration.

The inclusion criteria for the healthy subjects were; no history of liver disease, normal aspect of the liver and the spleen on ultrasound examination and LS values obtained by $\mathrm{TE}<7 \mathrm{kPa}$. The healthy volunteers were 29 medical students and staff members (nurses and medical doctors), who are regularly tested and screened for $\mathrm{HCV} / \mathrm{HBV}$. The remaining 27 were patients from other departments, with normal liver function tests, negative for HCV/HBV infections, without severe cardiac disease or heart failure.

The inclusion criteria for patients with chronic liver disease were: known chronic viral (B and $\mathrm{C}$ viruses) or nonviral hepatitis (alcoholic or non-alcoholic steatohepatitis, primary biliary cirrhosis) or cirrhosis; aminotranferases levels $<3 x$ normal values. We excluded patients undergoing antiviral treatment, patients with ascites, signs of biliary obstruction and liver congestion secondary to heart failure, and patients with focal liver lesions. We obtained liver stiffness (LS) measurements from all subjects in the same session by means of TE and 2DSWE.GE. We excluded 28 patients because LS measurements obtained from TE or/and 2D-SWE.GE were unreliable; the remaining 303 patients were included in the final statistical analysis.

\section{Transient Elastography (TE)}

TE was performed with FibroScan ${ }^{\circledR}$ device (EchoSens, Paris, France) in fasting condition. In each patient, we aimed for 10 valid LS measurements. The examination was performed in supine position, by intercostal approach, with the right arm in maximum abduction, using the $\mathrm{M}$ probe (standard probe - transducer frequency 3.5 $\mathrm{MHz}$ ) or the XL probe (transducer frequency $2.5 \mathrm{MHz}$ ). 
In all patients, the $\mathrm{M}$ probe was used first, and if the results were unreliable we used the XL probe. A median value of 10 valid LS measurements was calculated and the results were expressed in kilopascals $(\mathrm{kPa})$. Reliable measurements were defined as: median value of 10 valid LS measurements with a success rate $(\mathrm{SR}=$ ratio of the number of successful acquisitions divided by the total number of acquisitions) $\geq 60 \%$ and an interquartile range interval (IQR $=$ the difference between the 75th and 25 th percentile, essentially the range of the middle $50 \%$ of the data) $<30 \%$ $[30,31]$. The operators who performed TE measurements had at least 2 years experience with the method (defined as more than 500 examinations) and were blinded to all clinical, biologic and 2D-SWE data.

To discriminate between stages of fibrosis, we used the cut-off values for TE calculated in the Tsochatzis meta-analysis, $\mathrm{F} \geq 2: 7 \mathrm{kPa} ; \mathrm{F} \geq 3: 9.5 \mathrm{kPa} ; \mathrm{F}=4: 12 \mathrm{kPa}$ [10].

\section{D-Shear Wave Elastography (2D-SWE)}

LS evaluation by 2D-SWE was performed using a LOGIQ E9 system (GE Healthcare, Chalfont St Giles, United Kingdom) (version 2.0). All measurements were performed in fasting condition, in supine position, with the right arm in maximum abduction, by intercostal approach, in the right liver lobe, in the best acquired acoustic window for liver evaluation. LS measurements using 2D-SWE.GE were performed using a C1-6-D convex probe. The SWE region-of-interest (ROI) was placed at least $1-2 \mathrm{~cm}$ below the liver capsule, in a region free of large vessels. Once a suitable image was found, the patient was asked to suspend breathing and afterwards image acquisition was initiated. Usually 2 or 3 colored image frames were acquired during 5 seconds of suspended breathing. The process was repeated until at least 10 shear wave frames were acquired. Within each saved SWE image and frame a circular measurement region was placed and the measurement obtained. The average stiffness, expressed in terms of Young's Modulus within each measurement region, was automatically recorded by the system in a worksheet. Ten measurement regions were typically placed on different shear wave image frames or at non-overlapping locations within the same frame so that 10 consecutive LS measurements were obtained from each subject. The system automatically calculated the median value and IQR of the valid measurements. A valid LS assessment was considered as the median value of 10 measurements acquired in an homogenous area with an IQR $<30 \%$ [32]. The results of LS measurements were expressed in $\mathrm{kPa}$, similar to TE. The operators who performed 2D-SWE.GE measurements were experienced in ultrasound and elastographic evaluations, and were blinded to all clinical, biological and other elastographic measurement data.

\section{Statistical analysis}

The statistical analysis was performed using MedCalc Software, version 12.5.0.0 (MedCalc program, Belgium) and SPSS, Version 20.0 (IBM Statistics). The Kolmogorov-Smirnov test was used for testing the distribution of numerical variables. The mean value and standard deviation were calculated for numerical variables with normal distribution, while in cases of non-normal distribution, median values and range intervals were used, whereas categorical variables were reported as the number (proportion) of patients with/without the specific characteristic. The Student's t-test was used for group comparisons of continuous variables with a normal distribution and nonparametric tests (the Mann-Whitney U-test was applied for variables with non-normal distribution). Group comparisons of categorical variables were performed using Pearson's $x^{2}$-test. To avoid overestimating the accuracy of measurement results and validate the model, we assessed the K-fold crossvalidation by taking random LS measurements in groups of five and partitioning them from our cohort [33].

Areas under receiver operating characteristic (AUROC) curves were calculated for 2D-SWE.GE values to identify discriminating cut-offs for various stages of liver fibrosis. The optimal cut-off values were determined from AUROC curve analysis, by using the Bayesian analysis, using the optimal criterion and avoiding the misclassification of true positives subjects. Positive predictive value (PPV - true positive cases/all positive cases), negative predictive value (NPV - true negative cases/all negative cases) and diagnostic accuracy (sum of true positive and true negative cases/total number of cases) were calculated [34]. Ninety-five percent confidence intervals were calculated for each predictive test and a p-value $<0.05$ was considered to reveal statistical significance.

\section{Results}

Liver stiffness was evaluated by means of TE and 2D-SWE.GE in 331 patients. The main characteristics of the subjects included in the study are presented in Table I. The rate of reliable LS measurements was similar for TE and for 2D-SWE.GE: $94.2 \%(312 / 331)$ vs. $95.8 \%$ $(317 / 331), p=0.44$. From the valid TE measurements, $67.6 \%$ were performed using the $\mathrm{M}$ probe and $32.4 \%$ by using the XL probe. Higher BMI was significantly associated with the impossibility to obtain reliable LS measurements by means of TE and 2D-SWE.GE (Table II).

LS values obtained ranged from 3 to $22.5 \mathrm{kPa}$ for 2D-SWE.GE, and from 2.5 to $75 \mathrm{kPa}$ for TE. Mean liver stiffness values assessed by 2D-SWE.GE were significantly lower than those obtained by TE (paired t-test): $10.14 \pm 4.24 \mathrm{kPa}$ vs. $16.72 \pm 13.4 \mathrm{kPa}(\mathrm{p}<0.0001)$ (fig 1$)$. 
Table I. Main characteristics of the study group.

\begin{tabular}{ll}
\hline Parameter & \\
\hline Age (years) & $55(19-85)$ \\
Gender & $\mathrm{n}=127(38.4 \%)$ \\
$\quad$ Male & $\mathrm{n}=204(61.6 \%)$ \\
$\quad$ Female & $27.3 \pm 5.3$ \\
BMI $\left(\mathrm{kg} / \mathrm{m}^{2}\right)$ & \\
Distribution of subjects by BMI: & $\mathrm{n}=9(2.7 \%)$ \\
$\quad$ Underweight $(\mathrm{BMI}<18.5 \mathrm{~kg} / \mathrm{m} 2)$ & $\mathrm{n}=113(34.1 \%)$ \\
$\quad$ Normal weight $(\mathrm{BMI}=18.5-24.9 \mathrm{~kg} / \mathrm{m} 2)$ & $\mathrm{n}=119(36 \%)$ \\
$\quad$ Overweight $(\mathrm{BMI}=25-29.9 \mathrm{~kg} / \mathrm{m} 2)$ & $\mathrm{n}=90(27.2 \%)$ \\
$\quad$ Obese (BMI $\geq 30 \mathrm{~kg} / \mathrm{m} 2)$ & $\mathrm{n}=56(17 \%)$ \\
Diagnosis: & $\mathrm{n}=216(65.2 \%)$ \\
$\quad$ Healthy volunteers & $\mathrm{n}=40(12.1 \%)$ \\
$\quad$ Chronic hepatitis C (HCV) & $\mathrm{n}=19(5.7 \%)$ \\
$\quad$ Chronic hepatitis B (HBV) & \\
$\quad$ Chronic non - viral hepatitis (NAFLD, alcoholic, PBC) &
\end{tabular}

Numerical variables with normal distribution are presented as mean value \pm standard deviation, while variables with non-normal distribution are presented as median values and range intervals.

$\mathrm{n}$ - number of patients; NAFLD - non-alcoholic fatty liver disease; PBC - primary biliary cirrhosis; BMI - Body Mass Index

Table II. Factors associated with the impossibility to obtain reliable liver stiffness measurements by TE and 2D-SWE.GE.

\begin{tabular}{|c|c|c|c|}
\hline Parameter & Patients with reliable LS measurements & Patients without reliable LS measurements & P - Value \\
\hline \multicolumn{4}{|c|}{ Transient Elastography (TE) } \\
\hline Age (years) & $54 \pm 13.7$ & $60 \pm 10.3$ & 0.06 \\
\hline Gender Male & $\mathrm{n}=121(38.8 \%)$ & $\mathrm{n}=6(31.6 \%)$ & 0.7 \\
\hline Female & $\mathrm{n}=191(61.2 \%)$ & $\mathrm{n}=13(68.4 \%)$ & 0.7 \\
\hline $\operatorname{BMI}\left(\mathrm{kg} / \mathrm{m}^{2}\right)$ & $27.2 \pm 5.2$ & $30.2 \pm 6.3$ & 0.021 \\
\hline \multicolumn{4}{|l|}{ 2D-SWE.GE } \\
\hline Age (years) & $54 \pm 13.9$ & $61 \pm 6$ & 0.06 \\
\hline GenderMale & $\mathrm{n}=121(38.2 \%)$ & $\mathrm{n}=6(42.8)$ & 0.94 \\
\hline Female & $\mathrm{n}=196(61.8 \%)$ & $\mathrm{n}=8(57.2 \%)$ & 0.94 \\
\hline BMI $\left(\mathrm{kg} / \mathrm{m}^{2}\right)$ & $27.2 \pm 5.2$ & $32.8 \pm 4.4$ & 0.0001 \\
\hline
\end{tabular}

Numerical variables with normal distribution are presented as mean value \pm standard deviation, while variables with non-normal distribution are presented as median values and range. BMI - body mass index.

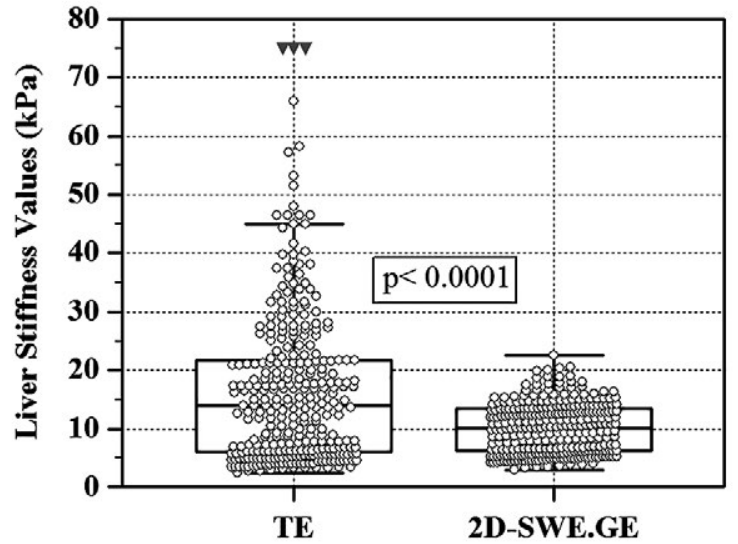

Fig 1. Mean LS values assessed by means of TE and 2D-SWE. GE

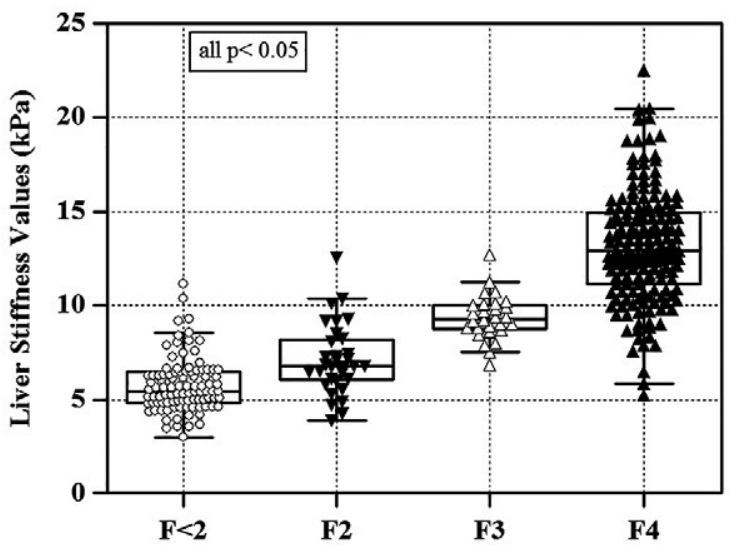

Fig 2. Mean liver stiffness values assessed by 2D-SWE.GE for different stages of fibrosis using TE as the reference 
Table III. Predictive values of liver stiffness evaluated by means of 2D-SWE for various stages of fibrosis, using TE as reference the method.

\begin{tabular}{llllllll}
\hline $\begin{array}{l}\text { Fibrosis } \\
\text { stage }\end{array}$ & $\begin{array}{l}\text { 2D-SWE.GE } \\
\text { cut-off }(\mathbf{k P a})\end{array}$ & AUROC & Sensitivity (\%) & Specificity (\%) & $\begin{array}{l}\text { PPV } \\
(\%)\end{array}$ & $\begin{array}{l}\text { NPV } \\
(\%)\end{array}$ & $\begin{array}{l}\text { Accura- } \\
\text { cy (\%) }\end{array}$ \\
\hline $\mathrm{F} \geq 2$ & $>6.7 \mathrm{CI}(6.5-8.5)$ & $0.95 \mathrm{CI}(0.92-0.97)$ & $92.7 \mathrm{CI}(88.3-95.9)$ & $85.5 \mathrm{CI}(77-91.9)$ & $93.2 \mathrm{CI}(88.8-96.2)$ & $84.7 \mathrm{CI}(76-91.2)$ & 90.4 \\
$\mathrm{~F} \geq 3$ & $>8.2 \mathrm{CI}(7.16-9.2)$ & $0.97 \mathrm{CI}(0.96-0.99)$ & $95 \mathrm{CI}(90.8-97.7)$ & $89.3 \mathrm{CI}(82.5-94.2)$ & $93 \mathrm{CI}(88.3-96.2)$ & $92.4 \mathrm{CI}(86-96.4)$ & 92.7 \\
$\mathrm{~F}=4$ & $>9.3 \mathrm{CI}(9.2-10.1)$ & $0.96 \mathrm{CI}(0.95-0.98)$ & $91.7 \mathrm{CI}(86.5-95.4)$ & $92.5 \mathrm{CI}(86.7-96.4)$ & $93.9 \mathrm{CI}(89.1-97.1)$ & $89.9 \mathrm{CI}(83.6-94.3)$ & 92 \\
\hline
\end{tabular}

F - fibrosis; AUROC - area under a receiver operating curve; PPV - positive predictive value; NPV - negative predictive value.

Reliable liver stiffness measurements were obtained with both methods (TE and 2D-SWE.GE) in 303/331 $(91.5 \%)$ subjects. Twenty eight $(8.5 \%)$ subjects were excluded because unreliable measurements were obtained from either TE and/or 2D-SWE.GE. Considering TE as the reference method to differentiate between stages of fibrosis, the distribution of liver fibrosis using the TE cut-offs proposed by the Tsochatzis meta-analysis was: $\mathrm{F}<2: 91 / 303$ (30.1\%); F=2: $31 / 303$ (10.2\%); F=3: 37/303 (12.2\%); $\mathrm{F}=4: 144 / 303$ (47.5\%).

The mean values of LS measurements by 2D-SWE. GE for various stages of liver fibrosis as assessed by TE were: for $\mathrm{F}<2$ : $5.7 \pm 1.4 \mathrm{kPa}$; for $\mathrm{F} 2: 7.1 \pm 2.1 \mathrm{kPa}$; for $\mathrm{F} 3$ : $9.4 \pm 1.7 \mathrm{kPa}$; for F4: $13.1 \pm 2.9 \mathrm{kPa}$ (fig 2).

A direct, strong correlation $(\mathrm{r}=0.83)$ was observed between LS values assessed by means of TE elastography and by 2 D-SWE.GE $(\mathrm{p}<0.0001)$. The K-fold cross validation confirmed that the measurement's results accuracy was not overestimated. Considering TE as the reference method, we calculated the performance of 2D-SWE.GE as well as the cut-off values for diagnosing significant fibrosis $(F \geq 2)$, severe fibrosis $(F \geq 3)$ and cirrhosis $(F=4)$ (Table III).

The group of healthy volunteers consisted of $56 \mathrm{sub}-$ jects without known liver pathology, with the mean age of $38 \pm 15.9(20-85), 36(64.3 \%)$ female and $20(35.7 \%)$ male. All 56 patients had valid measurements with both techniques. The mean LS values determined by $2 \mathrm{D}$ SWE.GE in healthy volunteers were $5.3 \pm 1.3 \mathrm{kPa}$ and $4.75 \pm 0.94 \mathrm{kPa}$ for TE.

\section{Discussions}

This study is to our knowledge the first that evaluates the performance of this novel elastographic method - 2D-SWE.GE, in terms of feasibility and performance for predicting different stages of liver fibrosis, other than the manufacturer's recommendations [32].

We found that the rate of reliable LS measurements was similar for the two methods, TE and 2D-SWE.GE (94.2\% vs. 95.8\%). For both methods, higher BMI was associated with the impossibility to obtain reliable LS measurements. It is known from previous studies that higher BMI can lead to failed or unreliable results for TE $[31,35]$. Furthermore, studies have shown that for 2D-SWE techniques, it is more difficult to obtain reliable results for obese and overweight subjects [28]. Narrow intercostal spaces can lead to invalid LS measurements, and represents a limitation of the 2D-SWE technique [36,37]. Nevertheless, there is no published data regarding limitations of the 2D-SWE.GE.

When assessing the recommendations proposed by the manufacturer regarding the use of quality parameters (10 valid measurements and $\mathrm{IQR}<30 \%$ ) [32], the feasibility of 2D-SWE.GE was very good. Concerning the feasibility of other 2D-SWE techniques, according to published data, three or five LS measurements by 2D-SWE.SSI can be obtained in $90-98.9 \%$ of cases $[28,29,38,39]$, but if quality parameters are applied (IQR $<30 \%$ and $\mathrm{SR} \geq 60 \%$ ), the rate of reliable measurements can decrease to $71.3 \%$ [40].

We compared LS measurements by 2D-SWE.GE to those obtained by TE considered as reference method in $91.5 \%$ of the subjects from the study group, who had reliable measurements by means of both elastographic methods. A highly significant correlation was found between the two elastographic methods $(r=0.83)$, but the LS values obtained by means of 2D-SWE.GE were significantly lower than those obtained by TE.

We calculated the LS cut-off values by 2 D-SWE.GE for predicting significant fibrosis, severe fibrosis and cirrhosis, considering this a strong point of our study, since there are no published studies in this regard, besides the manufacturers' recommendations $(\mathrm{F}>0-5.48 \mathrm{kPa} ; \mathrm{F}>1$ $8.29 \mathrm{kPa} ; \mathrm{F}>2-9.4 \mathrm{kPa} ; \mathrm{F}>3-11.9 \mathrm{kPa})$ [32]. We found cut-off values for predicting significant fibrosis, severe fibrosis, and cirrhosis lower than those recommended by the manufacturer [32]. These differences could be explained by the fact that the preliminary study included a small number of subjects (85) in comparison with our study (331), furthermore the initial study was performed with the first released elastographic software (version 
1.0 ), while the present one was performed using the upgraded elastographic module (version 2.0).

An advantage of 2D-SWE techniques in comparison with TE is that they are guided by B-mode imaging, thus being both color-coded and numeric methods. Using the color-coded map, the operator can choose to place the ROI in which LS is to be measured in the most homogenous area, and use the color homogeneity as a qualitative criterion for the evaluation. The advantage of real-time methods (2D-SWE) is the visual inspection of the liver during LS measurements so that it allows avoiding large vessels and placing the measuring box far enough from the liver capsule (more than $1 \mathrm{~cm}$ ), to avoid interference.

Regarding LS measurements by 2D-SWE.GE in healthy subjects, there are no current published data. In our study the mean LS value in 56 healthy subjects was $5.3 \mathrm{kPa}$.

Although our study included a large number of subjects evaluated by means of TE and 2D-SWE.GE, it does present some limitations. The main limit of our study is the absence of liver biopsy as the "gold standard", but our reference method was TE, which is a validated method in the daily practice. Furthermore, our cohort included patients with a heterogeneous etiology of liver disease. However, the majority of subjects included were $\mathrm{HCV}$ positive $(65 \%)$, so that this study could be considered as a starting point to use 2D-SWE.GE in the assessment of liver fibrosis in chronic hepatitis C patients. Also, using the Boursier criteria for LS measurements by means of TE could increase the method's feasibility, but they were not used in our study because they are not recommended at this time in international guidelines. Finally, viral markers or biochemical liver enzymes were not performed for all the healthy subjects, as 29 of the volunteers were medical students or staff who are regularly screened for $\mathrm{HCV} / \mathrm{HBV}$, but they all fulfilled the inclusion criteria and did not have any known liver disease or history of it.

In order to establish more accurate LS cut-off values for predicting various stages of liver fibrosis by 2DSWE.GE, further large multicentre studies are needed, preferably using liver biopsy as the reference method.

In conclusion, we found that 2D-SWE.GE is a reliable method for non-invasive evaluation of liver fibrosis. LS values obtained by means of 2D-SWE.GE were significantly lower than those obtained by TE. Higher BMI was associated with the impossibility to obtain reliable LS measurements for both TE and 2D-SWE.GE. The best $2 D$-SWE.GE cut-off values for predicting $F \geq 2, F \geq 3$ and $\mathrm{F}=4$ were $6.7 \mathrm{kPa}, 8.2 \mathrm{kPa}$ and $9.3 \mathrm{kPa}$, respectively.

Conflict of interest: none

\section{References}

1. Gebo KA, Herlong HF, Torbenson MS, et al. Role of liver biopsy in management of chronic hepatitis $\mathrm{C}$ : a systematic review. Hepatology 2002;36:S161-S172.

2. Seeff LB, Everson GT, Morgan TR, et al. Complication rate of percutaneous liver biopsies among persons with advanced chronic liver disease in the HALT-C trial. Clin Gastroenterol Hepatol 2010;8:877-883.

3. Regev A, Berho M, Jeffers LJ, et al. Sampling error and intraobserver variation in liver biopsy in patients with chronic HCV infection. Am J Gastroenterol 2002;97:2614-2618.

4. Bedossa P, Dargère D, Paradis V. Sampling variability of liver fibrosis in chronic hepatitis C. Hepatology 2003;38:1449-1457.

5 . Sporea I. Is there a real future for liver elastography? J Gastrointestin Liver Dis 2012; 21:129-131.

6. Poynard T, Imbert-Bismut F, Ratziu V, et al. Biochemical markers of liver fibrosis in patients infected by hepatitis $\mathrm{C}$ virus: longitudinal validation in a randomized trial. J Viral Hepat 2002;9:128-133.

7. Cosgrove D, Piscaglia F, Bamber J, et al. EFSUMB guidelines and recommendations on the clinical use of ultrasound elastography. Part 2: Clinical applications. Ultraschall Med 2013;34:238-253.

8. Sporea I. One or more elastographic methods for liver fibrosis assessment? Med Ultrason 2015;17:137-138.

9. European Association for Study of Liver; Asociacion Latinoamericana para el Estudio del Higado. EASL-ALEH Clinical Practice Guidelines: Non-invasive tests for evaluation of liver disease severity and prognosis. J Hepatol 2015;63:237-264.

10. Tsochatzis EA, Gurusamy KS, Ntaoula S, Cholongitas E, Davidson BR, Burroughs AK. Elastography for the diagnosis of severity of fibrosis in chronic liver disease: a metaanalysis of diagnostic accuracy. J Hepatol 2011;54:650659.

11. Friedrich-Rust M, Ong MF, Martens S, et al. Performance of transient elastography for the staging of liver fibrosis: a meta-analysis. Gastroenterology 2008;134:960-974.

12. Castéra L,Vergniol J, Foucher J, et al. Prospective comparison of transient elastography, Fibrotest, APRI, and liver biopsy for the assessment of fibrosis in chronic hepatitis C. Gastroenterology 2005;128:343-350.

13. European Association for the Study of the Liver. EASL Recommendations on Treatment of Hepatitis C 2016. J Hepatol 2017;666:153-194.

14. European Association For The Study Of The Liver. EASL clinical practice guidelines: Management of chronic hepatitis B virus infection. J Hepatol 2012;57:167-185.

15. Ziol M, Handra-Luca A, Kettaneh A, et al. Noninvasive assessment of liver fibrosis by measurement of stiffness in patients with chronic hepatitis C. Hepatology 2005;41:48-54.

16. Sporea I, Șirli R, Deleanu A, et al. Liver stiffness measurements in patients with HBV vs HCV chronic hepatitis: a comparative study. World J Gastroenterol 2010;16:48324837. 
17. Cardoso AC, Carvalho-Filho RJ, Stern C, et al. Direct comparison of diagnostic performance of transient elastography in patients with chronic hepatitis B and chronic hepatitis C. Liver Int 2012;32:612-621.

18. Wong VW, Vergniol J, Wong GL, et al. Diagnosis of fibrosis and cirrhosis using liver stiffness measurement in nonalcoholic fatty liver disease. Hepatology 2010;51:454-462.

19. Corpechot C, El Naggar A, Poujol-Robert A, et al. Assessment of biliary fibrosis by transient eleastography in patients with PBC and PSC. Hepatology 2006;43:1118-1124.

20. Donato MF, Rigamonti C, Colombo M, Can protocol liver biopsy be avoided to evaluate post-transplant hepatitis $\mathrm{C}$ recurrence? Transient elastography makes it possible. Dig Liver Dis 2010;42:307.

21. Șirli R, Sporea I, Bota S, Jurchiș A. Factors influencing reliability of liver stiffness measurements using transient elastography (M-probe)-monocentric experience. Eur J Radiol 2013;82:e313-e316.

22. Şirli R, Sporea I, Deleanu A et al. Comparison between the $\mathrm{M}$ and XL probes for liver fibrosis assessment by transient elastography. Med Ultrason 2014;16:119-122.

23. de Lédinghen V, Vergniol J, Foucher J, El-Hajbi F, Merrouche W, Rigalleau V. Feasibility of liver transient elastography with FibroScan using a new probe for obese patients. Liver Int 2010;30:1043-1048.

24. Coco B, Oliveri F, Maina AM, et al. Transient elastography:a new surrogate marker of liver fibrosis influenced by major changes of transaminases. J Viral Hepat 2007;14:360-369.

25. Millonig G, Reimann FM, Friedrich S, et al. Extrahepatic cholestasis increases liver stiffness (FibroScan) irrespective of fibrosis. Hepatology 2008;48:1718-1723.

26. Colli A, Pozzoni P, Berzuini A, et al. Decompensated chronic heart failure: increased liver stiffness measured by means of transient elastography. Radiology 2010;257:872878.

27. Sporea I, Bota S, Gradinaru-Taşcău O, Sirli R, Popescu A, Jurchiş A. Which are the cut-off values of 2D-Shear Wave Elastography (2D-SWE) liver stiffness measurements predicting different stages of liver fibrosis, considering Transient Elastography (TE) as the reference method? Eur J Radiol 2014;83:e118-e122.

28. Ferraioli G, Tinelli C, Dal Bello B, et al; Liver Fibrosis Study Group. Accuracy of real-time shear wave elastogra- phy for assessing liver fibrosis in chronic hepatitis $\mathrm{C}$ : a pilot study. Hepatology 2012;56:2125-2133.

29. Leung VY, Shen J, Wong VW, et al. Quantitative elastography of liver fibrosis and spleen stiffness in chronic hepatitis B carriers: comparison of shear-wave elastography and transient elastography with liver biopsy correlation. Radiology 2013;269:910-918.

30. Sandrin L, Fourquet B, Hasquenoph JM, et al. Transient elastography: a new noninvasive method for assessment of hepatic fibrosis. Ultrasound Med Biol 2003;29:1705-1713.

31. Castera L, Foucher J, Bernard PH, et al. Pitfalls of liver stiffness measurement: a 5-year prospective study of 13,369 examinations. Hepatology 2010;51:828-835.

32. GE Healthcare LOGIQ E9 Shear Wave Elastography Whitepaper recommendations. Available at: http://www3. gehealthcare.com.

33. Ross KA, Jensen CS, Snodgrass R, et al. Cross-Validation. In: Encyclopedia of Database Systems. Springer US, Boston, MA, 2009:532-538. doi:10.1007/978-0-387-39940-9_565.

34. Hanley JA, McNeil BJ. The meaning and use of the area under a receiver operating characteristic (ROC) curve. Radiology 1982;143:29-36.

35. Sporea I, Sirli R, Mare R, Popescu A, Ivascu SC. Feasibility of Transient Elastography with M and XL probes in real life. Med Ultrason 2016;18:7-10.

36. Grădinaru-Taşcău O, Sporea I, Bota S, et al. Does experience play a role in the ability to perform liver stiffness measurements by means of supersonic shear imaging (SSI)? Med Ultrason 2013;15:180-183.

37. de Lédinghen V, Vergniol J. Transient elastography (FibroScan). Gastroenterol Clin Biol 2008;32(6 Suppl 1):58-67.

38. Hudson JM, Milot L, Parry C, Williams R, Burns PN. Inter- and intra-operator reliability and repeatability of shear wave elastography in the liver: a study in healthy volunteers. Ultrasound Med Biol 2013;39:950-955.

39. Poynard T, Munteanu M, Luckina E, et al. Liver fibrosis evaluation using real-time shear wave elastography: applicability and diagnostic performance using methods without a gold standard. J Hepatol 2013;58:928-935.

40. Sporea I, Bota S, Jurchis A, et al. Acoustic radiation force impulse and supersonic shear imaging versus transient elastography for liver fibrosis assessment. Ultrasound Med Biol 2013;39:1933-1941. 